\title{
In silico modeling of immunotherapy and stroma-targeting therapies in human colorectal cancer
}

Jakob Nikolas Kather ${ }^{1,2,3}$, Jan Poleszczuk ${ }^{4}$, Meggy Suarez-Carmona ${ }^{1,3}$, Johannes Krisam ${ }^{5}$, Pornpimol Charoentong ${ }^{1,3}$, Nektarios A. Valous ${ }^{1,3}$, Cleo-Aron Weis ${ }^{6}$, Luca Tavernar ${ }^{7,8}$, Florian Leiss $^{9}$, Esther Herpel ${ }^{7,8}$, Fee Klupp ${ }^{10}$, Alexis Ulrich ${ }^{10}$, Martin Schneider ${ }^{10}$, Alexander Marx ${ }^{6}$, Dirk Jäger ${ }^{1,2,3}$, Niels Halama ${ }^{1,2,3, *}$

${ }^{1}$ Department of Medical Oncology and Internal Medicine VI, National Center for Tumor Diseases, University Hospital Heidelberg, Heidelberg, Germany

${ }^{2}$ German Cancer Consortium (DKTK), Heidelberg, Germany

${ }^{3}$ Applied Tumor Immunity, German Cancer Research Center (DKFZ), Heidelberg, Germany

${ }^{4}$ Nalecz Institute of Biocybernetics and Biomedical Engineering, Polish Academy of Sciences, Warsaw, Poland

${ }^{5}$ Institute of Medical Biometry and Informatics, University Hospital Heidelberg, Heidelberg, Germany

${ }^{6}$ Department of Pathology, University Medical Center Mannheim, Mannheim, Germany

${ }^{7}$ Institute of Pathology, Heidelberg University, Heidelberg, Germany

${ }^{8}$ Tissue Bank of the National Center for Tumor Diseases (NCT) Heidelberg, Germany

${ }^{9}$ Definiens AG, Munich, Germany

${ }^{10}$ Department of Surgery, University Hospital Heidelberg, Heidelberg, Germany 


\section{* Correspondence to}

Niels Halama, MD

Medical Oncology, National Center for Tumor Diseases (NCT)

Im Neuenheimer Feld 460

69120 Heidelberg, Germany

niels.halama@nct-heidelberg.de

$+4962215638397$

Running Title: Immune cell - stroma interactions in colorectal cancer

Keywords: Cancer immunotherapy, Tumor microenvironment, Stroma, Colorectal Cancer, Modeling

\section{Financial Support}

JN Kather is supported by the "Heidelberg School of Oncology" (NCT-HSO) and by the "German Consortium for Translational Cancer Research" (DKTK) fellowship program. A Marx is supported by a grant of the German Federal Ministry of Education and Research (BMBF) within the Framework of the Research Campus M²oBITE (grant 13GW0091E). 


\section{Abstract}

Despite the fact that the local immunological microenvironment shapes the prognosis of colorectal cancer, immunotherapy has shown no benefit for the vast majority of colorectal cancer patients. A better understanding of the complex immunological interplay within the microenvironment is required. In this study, we utilized wet lab migration experiments and quantitative histological data of human colorectal cancer tissue samples $(n=20)$ including tumor cells, lymphocytes, stroma and necrosis to generate a multi-agent spatial model. The resulting data accurately reflected a wide range of situations of successful and failed immune surveillance. Validation of simulated tissue outcomes on an independent set of human colorectal cancer specimens $(n=37)$ revealed the model recapitulated the spatial layout typically found in human tumors. Stroma slowed down tumor growth in a lymphocyte-deprived environment but promoted immune escape in a lymphocyte-enriched environment. A subgroup of tumors with less stroma and high numbers of immune cells showed high rates of tumor control. These findings were validated using data from colorectal cancer patients $(n=261)$. Low-density stroma and high lymphocyte levels showed increased overall survival (hazard ratio $0.322, p=0.0219$ ) as compared with high stroma and low lymphocyte levels. To guide immunotherapy in colorectal cancer, simulation of immunotherapy in pre-established tumors showed that a complex landscape with optimal stroma permeabilization and immune cell activation is able to markedly increase therapy response in silico. These results can help guide the rational design of complex therapeutic interventions which target the colorectal cancer microenvironment.

Major Findings (nontechnical description): We present a computer-based model of lymphocyte - tumor - stroma interactions. This model reproduces key aspects of human colorectal cancer tissue, predicts survival in an independent patient cohort and proposes a new strategy for successful immunotherapy in colorectal cancer. 


\section{Quick Guide to Equations and Assumptions}

Our model is based on a minimal set of assumptions that are backed by our own data or previously published data. Two types of agents are modeled: tumor cell agents and immune cell agents. Immune cells in our model represent T-lymphocytes, whose main fraction are cytotoxic lymphocytes. All assumptions for the model are made explicit in Table 1 and all model parameters are listed in Suppl. Table S1. Each agent occupies exactly one position on a twodimensional rectangular grid with dimensions $\mathrm{N} \times \mathrm{M}$ and Moore neighborhood (each grid cell has eight neighbors). Only one agent can occupy a grid cell. Unlike in other agent-based models, all agents occupy the same grid and thus compete for space. This is thought to reflect the nature of colorectal cancer tissue which typically consists of densely packed cells. For simplicity, we refer to tumor cell agents as "tumor cells" and to immune cell agents as "immune cells". Upon initialization, one tumor cell is placed in the middle of the domain. Immune cells randomly appear each round (constant rate of influx). Then, successively, tumor cells and immune cells can act as shown in Figure 1A. Typically, 500 to 5000 model iterations (rounds) are performed after which complex spatial patterns can be observed (Figure 1B). In each iteration, each tumor cell randomly performs an action, with the following probabilities: Tupdeath for dying, Tupmig for migrating to a randomly picked free adjacent position, Tupprol for proliferating. All remaining cells will idle. If a tumor stem cell (stemness true) proliferates, it will generate an identical descendant with probability Tups. Otherwise, it will generate a descendant with no stemness. After tumor cells have acted, immune cells will act. With probabilities Impdeath, Impmig and Impprol they will die, migrate or proliferate. Immune cells do not have a stemness property. Sustained immune cell activation can give rise to stroma (fibrosis) through a desmoplastic reaction. Tumor cells and immune cells can be present in fibrotic areas. However, by default, they cannot move here (no permeability). The permeability of fibrotic (stromal) areas can be adjusted between 0 and 1 through the parameter stromaPerm. Tumor cells may die and seed necrosis with probability probSeedNecr. Necrosis is more likely to occur in the tumor center as its occurrence linearly scales with the distance from the smoothed tumor edge. From here on, we use the term "stroma" to refer to the tissue generated by fibrosis through exhausted immune cells. 
Although in reality, stroma might also arise through inflammation-independent mechanisms, we restrict our model on stroma induced by ongoing inflammation. To rule out early spontaneous tumor death, we required the tumor to be alive at least 50 iterations (or longer, if declared otherwise - up to four attempts per experimental run). The time scale of all events is scaled or adjusted through intermediary steps in such a way that one main iteration represents 12 hours. The resulting agent-based model shows emergent behavior and yields spatial patterns closely resembling those in histological samples of human colorectal cancer.

\section{Introduction}

In recent years, tumor immunotherapy has become available to treat malignant tumors with several drugs already approved for solid tumors. Their main mode of action is the activation of the adaptive immune system via checkpoint inhibition (1). Other widely used therapeutic strategies aim at increasing the number and the reactivity of effector cells by adoptive cell transfer or vaccination $(2,3)$. Additionally, many more drugs are currently investigated in clinical trials, especially combination therapies aiming at the stromal compartment $(4,5)$. These approaches act on the complex interactions in the tumor microenvironment, as we could show recently in macrophage-targeted immunotherapies (6).

Still, for most patients with solid tumors, no effective immunotherapy strategy is available. Especially for microsatellite-stable colorectal cancer, the most common form of this disease, immunotherapy has been largely ineffective. With a five-year survival of just $11 \%$ in metastatic diseases (7) and a huge disease burden (8), the therapeutic need is high. In this setting, the development of more complex interventions into the immune landscape requires detailed knowledge of the interactions of relevant players and their response to interventions. Although our understanding of these processes has advanced considerably in the last years, there is still no comprehensive systems perspective of all relevant interactions. To understand a complex system, it is not sufficient to have a detailed characterization of all its components. Instead, a complex system can show emergent behavior that does not arise from a specific component but from the interaction of different components. 
Agent-based models are a powerful method to investigate the interactions in complex systems (9). An agent is the smallest unit in this model and can show different types of stochastic behavior, including interaction with other agents. Although these models simplify many aspects of reality, they have been shown to be extremely useful in a wide number of circumstances (1012). In cancer research, these models are emerging as valuable tools to study emergent behavior in complex ecosystems (13), especially in stem-cell models of tumor growth $(14,15)$ and are used to study the mutational landscape of solid tumors $(16,17)$. Furthermore, they are increasingly used to optimize therapies, for example radiation therapy of solid tumors (18). Also, some models of immune-cell interactions with (19-24) or without tumor cells (25) have been proposed. Although these studies gave important insight into parts of the tumor-immune interaction, they did not accurately reproduce the diverse spatial patterns in human tumors and did not investigate therapeutic strategies.

In the present study, we generated a multi-agent-based model from quantitative histological and other wet lab data, based on the concept of immune surveillance (26). We focused on parameters that could be morphologically measured and created a simplified yet powerful model of cellular interactions that shows emergent behavior. This agent-based model incorporates stochastic interactions between tumor cells, immune cells and stroma and faithfully represents diverse spatial pattern observed in histological samples of human colorectal cancer tissue. Furthermore, the derived clinical predictions could be validated in an independent colorectal cancer cohort. This model was then used to systematically test the effect of different therapeutic interventions on this system and to create specific recommendations for effective immunotherapies.

\section{Materials and Methods}

\section{Ethics statement and tissue samples}

All experiments were conducted in accordance with the Declaration of Helsinki, the International Ethical Guidelines for Biomedical Research Involving Human Subjects (CIOMS), the Belmont Report and the U.S. Common Rule. N=20 human tissue samples of colorectal adenocarci- 
noma were used as a calibration cohort. These samples were retrieved from the pathology archive at UMM (University Medical Center Mannheim, Heidelberg University, Mannheim, Germany) after approval by the institutional ethics board (Ethics Board II at University Medical Center Mannheim, decision number 2017-806R-MA, granted to AM and waiving the need for informed consent for this retrospective and fully anonymized analysis of archival samples; Suppl. Table S2).

Another set of 37 colorectal adenocarcinoma samples was used as a morphological validation cohort, composed as follows: $\mathrm{N}=22$ tissue samples were provided by the tissue bank of the $\mathrm{Na-}$ tional Center for Tumor diseases (NCT, Heidelberg, Germany) in accordance with the regulations of the tissue bank and the approval of the ethics committee of Heidelberg University (tissue bank decision number 2152, granted to NH and JNK; informed consent was obtained from all patients as part of the NCT tissue bank protocol; Suppl. Table S3). N=15 additional samples was used as described before (Suppl. Table S3) (27). As the model was designed to be valid for all types of colorectal cancer tissues, we included primary tumor samples and colorectal cancer liver metastases in all cohorts (Suppl. Table S2 and S3).

\section{$\underline{\text { Histological assessment }}$}

We performed histological staining for Ki67 (Dako M7240 antibody, 1:100), active Caspase 3 (Abcam ab2302 antibody, 1:50) and CD3 (Leica Novocastra NCL-L-PS1 antibody, 1:50) on a Leica Bond automatic staining device using a hematoxylin-diaminobenzidine (DAB) staining protocol as described previously $(6,28)$. Stained whole slide tissue sections were digitized as described previously $(6,28)$. On histological sections, we manually identified areas homogenously occupied by tumor cells or immune cells (in the tumor or around the tumor). The fraction of Ki67positive cells (active Caspase 3 positive cells, respectively) was quantified in these regions of interest (ROI) per slide using a digital pathology approach analogous to our previously published approaches (29,30). Definiens Tissue Studio (Definiens AG, Munich, Germany) was used for semi-automatic tissue segmentation and automatic cell segmentation. The fraction of positive cells was calculated as the number of positively (diaminobenzidine-positive) stained cells divided by the number of all cells in the respective ROI. On average, each ROI contained approx- 
imately 3000 cells. Intensity thresholds for cell detection and classification were set manually and were identical for all images. The quality of cell segmentation was checked manually for each image and was found to be sufficient. We assumed that the median Ki67-positive fraction and the median active Caspase 3-positive fraction approximated the probability of a given cell type to proliferate or die at one time point. All raw measurements are listed in the Suppl. Data.

\section{Horizontal migration experiments on lymphocytes in vitro}

For the horizontal migration experiments, Matrigel (undiluted, BD, Germany) was evenly plated at the sides of a 24-well chamber in two half-moon shapes (cat eye configuration) and the remaining third in between was filled with either pure collagen or Matrigel with CXCL9 $(10 \mathrm{ng} / \mathrm{ml})$ and CXCL10 (10 ng/ml). After gelling overnight in a humid chamber at $37^{\circ} \mathrm{C}, 5 \% \mathrm{CO} 2$, the well was filled with a thin layer of T cell culture media and healthy donor T lymphocytes (CD3/CD28 activated and in an independent experiment non-activated) were placed on the right half-moon shaped third. After migration for $48 \mathrm{~h}$ at $37{ }^{\circ} \mathrm{C}$ the resulting distribution in the well was documented and distances were documented and used for the multi-agent model system.

\section{Estimation of key parameters}

Initially, our model had 22 parameters, as shown in Suppl. Table S1. These parameters were based on a clear set of assumptions. Key assumptions based on previous studies are related the tendency of immune cells to migrate towards tumor cells (31-33), lymphocyte exhaustion $(23,34)$, desmoplastic reaction $(35,36)$ and stromal permeability $(37)$. All assumptions are listed in Table 1. Some of the model parameters had been estimated in previous studies. Specifically, this applies to the maximum proliferation capacity of non-stem cells (tumor cells and immune cells alike), which we set to 10 analogous to (14). Also, it was previously shown that the maximum number of kills a lymphocyte can deliver can be validly estimated as five (23). Of the remaining parameters, five were measured histologically: Tumor cell proliferation and apoptosis, immune cell proliferation and apoptosis and distance to necrosis (Suppl. Figure S1A-F). Thus, 12 free parameters remained and were set to biologically plausible values (Suppl. Table S1).

Of 20 tissue samples, 12 contained necrosis and the distance to necrosis from the outer tumor margin was measured at three locations, giving 36 distance values. Mean distance was 1.01 
$\mathrm{mm}$, standard deviation was $0.62 \mathrm{~mm}$ so that $90 \%$ of all necrotic areas occurred within approximately $2 \mathrm{~mm}$ (1.64 sigma, rounded). In the model, the occurrence of necrosis was determined by probSeedNecr and it was more likely to be located at the tumor core, with the probability linearly increasing from 0 to $2 \mathrm{~mm}$ from the outer tumor margin.

Regarding the scale of the model, we measured cell density of tumor cells in $\mathrm{N}=20$ histological samples. We found that tumor cells and immune cells occupy an area of $222.8 \mu \mathrm{m}^{2}$ (median value). Although tumor cells are larger than lymphocytes, the uniform grid required that one grid cell should accommodate one cell. This assumption yielded a rectangular grid cells size of $14.9 \mu \mathrm{m}$. Thus, the length of 67 grid cells correspond to one $\mathrm{mm}$.

\section{Time discretization}

The events in our model (cell proliferation, migration, death) do necessarily occur with the same rate at each iteration. To account for these temporal differences, we introduced intermediary steps. We assumed that the median Ki67-positive fraction $f k$ is equal to the probability of cell division $p k$ in one iteration. Median tumor cell proliferation fraction (as measured in $\mathrm{N}=20$ tissue samples) was approximately 0.5 (Suppl. Figure S1A) and a full cell cycle typically takes approximately 24 hours. Therefore, we set one full iteration of the model as 12 hours. To find out the probability of cell death in each iteration, we measured the fraction $f c$ of tumor cells and immune cells positively stained for active caspase 3 . The process of apoptosis induction to completion takes approximately three hours (38), just a quarter of the time step in our model. Therefore, the probability of cell death $p c$ in each iteration was scaled appropriately. Regarding immune cell movement, we assumed that tumor-infiltrating lymphocytes (immune cell agents) migrate with an average speed of approximately $2 \mu \mathrm{m} / \min (2880 \mu \mathrm{m} / 24 \mathrm{~h})$ (32). These data were also qualitatively validated by our in vitro experiments (data not shown). In our model, this corresponds to 97 grid cells / $12 \mathrm{~h}$ (97 grid cells / iteration). Therefore, we introduced intermediary steps and allowed immune cells to move up to 97 times per iteration. Lastly, we scale the tumor cell killing events: It has been shown that tumor cell killing by cytotoxic Tlymphocytes is initiated within minutes (39) but takes approximately six hours to complete in vitro (40) and in vivo (31). Accordingly, we required that a killing event keeps a lymphocyte en- 
gaged for six hours and only after this period the lymphocyte can kill again (if it is not exhausted). It has been shown that tumor cell killing might require several hits by lymphocytes (40). This was not explicitly modeled in our system. Instead, we assumed that the killing probability parameter IMpkill already included multiple hits.

\section{$\underline{\text { Outcome assessment }}$}

In our simulation runs, we let the tumors grow for a fixed number of iterations (nSteps) before performing changes of the parameters. After an additional number of steps addSteps, tumor cell number was compared to the baseline. We assessed the outcome analogously to the RECIST criteria (41) based on the number of tumor cells at T=nSteps+addSteps as compared with $\mathrm{T}=n$ Steps. Complete remission (CR) was equivalent to the eradication of all tumor cell, partial remission (PR) was a reduction in tumor cell number by at least $30 \%$, progressive disease (PD) was an increase of tumor cell number by at least $20 \%$ and stable disease (SD) described all other outcomes.

\section{Computational implementation}

All simulations were implemented in MATLAB $^{\circledR}$ (Mathworks, Natick, MA, USA) R2017a. Parts of the code were run in parallel with MATLAB's Parallel Processing Toolbox. All experiments were run on a standard workstation (Intel i7 Processor, 8 cores, 32 GB RAM, Microsoft Windows 10.1). Typically, computing speed was several hundred simulations per hour. We release all source codes for the agent-based model under an open-source license (http://dx.doi.org/10.5281/zenodo.853342).

\section{Clinical data (TCGA)}

To validate the predictions of the model, a clinical validation cohort of $\mathrm{N}=261$ colorectal adenocarcinoma (COAD) patients from the NIH (National Institutes of Health) The Cancer Genome Atlas (TCGA) collective was used (42). The data was downloaded via the TCGA Data Portal as described before (43). All TCGA samples that were included in the analysis are listed in Suppl. Table S4. 


\section{$\underline{\text { Survival analysis }}$}

To assess the association between overall survival, stroma, lymphocytes and their interaction, we performed a survival analysis of the TCGA collective. Cox proportional hazard models were fitted, including overall survival as a dependent variable, and lymphocytes (high/low), stroma (high/low), and the interaction of lymphocytes and stroma as fixed factors. Lymphocyte infiltration and stroma content of tumor tissue were part of the data tables available at the TCGA data portal. These variables had been manually measured by pathologists as part of the original TCGA data curation. Patients were split into low and high at the median. Due to its role as a potential confounder, TNM status was also included into the model as a fixed factor. Hazard ratios for effect estimates with corresponding 95\% confidence intervals, and p-values for hazard ratios and the interaction term were computed. P-values smaller than 0.05 were regarded as statistically significant. The analysis was carried out using SAS v9.4 (SAS Institute, Carey, NC, USA). Furthermore, a Kaplan-Meier plot displaying the product-limit survival estimates alongside the number of subjects at risk for each of the four strata was created.

\section{Results}

The model recapitulates major immune phenotypes of solid tumors

We propose a new agent-based model of tumor-immune cell interactions that is based on a minimal set of assumptions (Table 1) and parameters (Suppl. Table S1). In this model, we observed emergent behavior on different scales, particularly with regard to tumor tissue morphology. Generally, four types of immunological phenotypes can be distinguished histologically: hot tumors, cold tumors, immune excluded tumors (with an immune cell rim around the tumor) and tumors that have been (almost) completely eradicated by immune cells (44). Our agentbased model was able to reproduce all these spatial patterns as shown in Figure 2A-D. We concluded that the model is in principle able to model all relevant types of immune surveillance in solid tumors. 
The model faithfully represents spatial patterns compared with histological data

To compare our model with histological spatial patterns in an objective way CD3-stained colorectal cancer tissue samples were used. This analysis entailed all $\mathrm{N}=20$ tissue samples from the calibration cohort (Suppl. Table S2) and N=37 additional samples from an independent validation cohort from a different institution (Suppl. Table S3). We analyzed spatial features of tumor/stroma distribution and found that the spatial layout of CD3-positive lymphocytes was always part of the spectrum of cold tumors (Figure 2A), hot tumors (Figure 2B), immune excluded tumors (Figure 2C) or eradicated tumors (Figure 2D). Specifically, resulting tumor nodules showed varying degrees of fibrosis and necrosis, mirroring spatial patterns in histological samples. This is shown in Suppl. Figure S2 A-C for an immune-excluded non-necrotic tumor, in Suppl. Figure S2 D-F for a largely necrotic tumor and in Suppl. Figure S2 G-I for a cold tumor with stromal core. We conclude that based on a calibration cohort and a morphological validation cohort, the model reproduced spatial architecture of tumor cells, stroma and lymphocytes sufficiently.

$\underline{\text { Stroma deprivation enables tumor eradication in a lymphocyte-enriched environment in silico }}$

To better elucidate the emergent dynamics, the behavior of tumors under different environmental conditions were investigated. In particular, we investigated immunological dynamics (immune surveillance) of a typical tumor with varying tumor-stroma ratio and varying numbers of tumor-reactive lymphocytes. To this end, we simulated the growth of 50 tumors for 60 days. At this point, tumors had reached a size of close to 5000 cells. Then, the immune cell influx was strongly increased (immune boost) and the parameter for stroma induction was varied to generate tumors with different lymphocyte and stroma contents (Figure 3A). According to these variations, four types of host response were investigated: Low and high stroma (Stro) generation (fibrosis seeding) and low and high immune cell (Lym) number.

We observed that the groups showed a drastically different behavior: Tumors in the "Stro low, Lym low" group showed an unhindered, exponential growth (Figure 3B). In comparison, the growth in the "Stro high, Lym low" group was slower, but still steadily rising. As can be seen in Figure 3C, both "Lym low" groups presented largely with progressive disease (PD) states at six 
months (180 days) after baseline. In contrast, a higher number of lymphocytes (Lym high) led to a phenotype with restrained tumor growth, as expected. Specifically, in the subgroup "Stro high, Lym high", tumor size was constrained to under 10000 cells (Figure 3B and C). Surprisingly, the "Stro low, Lym high" group showed an altogether different behavior: after an initial increase in tumor mass (Figure 3B), inflowing immune cells regained control and completely eradicated the tumor in almost all simulation runs (Figure 3C, Suppl. Figure S3).

Clinical validation of the predicted immunological dynamics demonstrates a combined risk fac$\underline{\text { tor }}$

In the model, the sub-group of tumors "Stro low, Lym high" by far the most favorable outcome of all simulated tumors. Tumor eradication reproducibly occurred only in the "Stro low, Lym high" group and not in the "Stro high, Lym high" group. This suggests that a high number of lymphocytes can only successfully constrain tumor growth if there is little stroma in the tumor.

We validated this prediction by analyzing a cohort of $\mathrm{N}=261$ patients from the TCGA database based on publicly available records $(42,43)$. For all patients, a manual histopathological quantification of stroma and lymphocytes was available as well as clinical follow-up data. Patients were stratified into high and low stroma content and lymphocyte number at the median. The only sub-group with a significant overall survival benefit as compared to the other groups was "Stro low, Lym high" in comparison to "Stro high, Lym high" group, as assessed by our Cox proportional hazards model (hazard ratio 0.309 for overall survival $0.322, p=0.0219$, confidence interval 0.122 to 0.849 , Figure 4A), reflected also by Kaplan-Meier curves for all groups (Figure 4B) . "Stro" and "Lym" alone were no significant predictors of overall survival and neither were all other subgroups as shown in Figure 4A. The $p$-value for the interaction between "Lym" and "Stro" was $p=0.1192$. Taking into account that the interaction test is commonly subject to a very small power (45), $p=0.1192$ for an interaction test, even though not statistically significant, can be deemed as an indicator for a quite prominent interaction between "Lym" and "Stro".

\section{An optimal combination of immunotherapy and stroma-targeted therapy}

Having confirmed stroma as an important modulating factor in the in silico experimental setup, we simulated an immunotherapy together with stroma targeting therapy. A simple yet realistic 
way of simulating stroma-targeted therapy is to modify the permeability of stroma with regard to cell migration $(46,47)$. Therefore, we let tumors grow to a diameter of approximately $2 \mathrm{~mm}$, which was typically reached after 120 days (Figure 5A). Then, we simulated an "immune boost", increasing the number of immune cells 2-fold, 4-fold and 8-fold. Also, stroma was permeabilized with a factor of $4 \%, 8 \%$ and $16 \%$. In accordance with our previous results, we saw that non-permeable stroma inhibited a tumor eradication (Figure 5B).

In tumors with low stroma permeability and/or low lymphocyte numbers, we observed mostly tumor progression, characterized by four typical phenotypes: stroma acting as a physical barrier that protects the tumor (Suppl. Figure S4A), rapid tumor outgrowth of immune control (Suppl. Figure S4B), tumors breaking through a physical barrier (Suppl. Figure S4C) and excessive immune cell exhaustion (Suppl. Figure S4D).

Contrariwise, tumors with high stroma permeability and high lymphocyte numbers were successfully eradicated in $75 \%$ of all simulation runs (Figure 5B). There was a striking duality in the effect of stromal permeabilization: In a lymphocyte-deprived environment, permeabilizing stroma had an adverse effect and led to increased tumor progression (bottom row in Figure 5B). With highly permeable stroma, this adverse effect persisted also in 2-fold immune boosting and was only superseded by a strong immune boost of 4-fold.

From these in-silico experiments with clinical validation, we conclude that stroma, arising through post-inflammatory fibrosis, has a dual role in solid tumors: under usual conditions, it can mitigate tumor growth to a small degree. After a simulated immune boost, stroma provides a mechanism for immune escape. Only the permeabilization of stroma in combination with immunotherapy can lead to tumor regression in this model.

\section{Discussion}

Within the concept of tumor immune surveillance, tumor cells and immune cells are engaged in an ongoing battle (26). From a conceptional perspective, a tumor disease develops if tumor cells temporarily win this fight by means of immune evasion. In line with this, immunotherapy 
aims at activating the $\mathrm{T}$ cells and thereby leading to tumor eradication in some patients. For colorectal cancer (microsatellite stable tumors), so far, no immunotherapy has shown efficacy. Driven by the clinical need to better understand the processes of immune evasion and activation, we developed a computer-based model that allowed us to investigate the dynamics governing tumor-immune cell interaction on a systems level (Figure 1A). This model was generated utilizing quantitative histological and other wet lab data as well as data from previous studies (Suppl. Table S1).

The model was fitted to histological data in two ways: First, quantitative histological data from Ki67 and active Caspase 3 immunostainings was used to estimate the proportion of actively dividing and dying cells as well as the overall cell density. In a second step, $\mathrm{N}=37$ human tumor tissue samples were used to show that the naturally occurring spatial patterns could be reproduced by our model, even on a large spatial scale (Suppl. Figure S2A-I).

Using this validated in silico model, a consistent and highly relevant interaction between lymphocytes and stroma was discovered: In an immune-deprived environment, stroma restrains tumor growth and tumors with little stroma grow faster. In contrast, in an immune-cell enriched environment, stroma inhibits tumor cell killing thus, immune-cell rich tumors with low amounts of stroma are eradicated while immune-cell rich tumors with high amounts of stroma are not (Figure 3). This leads to a bivariate risk-factor model for colorectal cancer that was validated in two independent validation steps: a morphological validation in $n=57$ tissue samples (Figure 2A-D, Suppl. Figure S2A-I) and a clinical validation in a set of $n=261$ patients (Figure 4AB).

As a next step, immune cell numbers and stromal permeability were gradually varied (Figure 5B). Strikingly, a very consistent pattern was apparent: increasing the number of lymphocytes could only lead to tumor eradication if stromal permeability was also increased, in a dosedependent manner (Figure 6). Stromal permeability increase without lymphocyte-enrichment was detrimental and led to faster tumor progression (Figure 6). This has important clinical consequences: increasing stromal permeability might greatly enhance the effectiveness of immunotherapy, but can also be dangerous if the tumor microenvironment is lymphocyte-deprived. 
Currently, several experimental therapies in the clinic aim to target the stroma. Indeed, most prominent are approaches with enzymes that aim to enhance the permeability of stroma $(4,5,48)$. Our data show that these approaches can be very effective but can also have adverse effects need to be considered before clinical development of new substances. Thus, for future clinical trials, the balance between stroma-targeting interventions and lymphocyte-targeting interventions should be investigated at the pre-clinical stage. In-silico models such as our model could be a part of this pre-clinical pipeline.

The contribution of stroma reaction and inflammation in colorectal cancer has been investigated before, jointly for both factors $(49)$ or separately $(50,51)$. However, to our knowledge, we report for the first time a bivariate interaction effect of both risk factors and provide an explanation for this behavior through in silico experiments. Our findings regarding fibrosis are in line with previous observations in biological studies and explain previous observations. Pancreatic cancer, for example, typically has pronounced tumor fibrosis. It has been shown that reducing fibrosis in this cancer benefits the tumor and reduces survival in a mouse model (52). This behavior also arises in our model. Furthermore, in line with our simulations, it has been shown that tumors can use the stroma to mitigate the immune response (53). Regarding immunotherapy, it has been suggested that immune response against a tumor is more efficient if the immune cells also destroy the stroma (48). Our model provides a simple explanation for this behavior.

Like all models, our model is not an exact copy of reality but simplifies some key aspects of realworld tumors. For example, killing of tumor cells by immune cells is a purely stochastic process in our model. Fine-tuning through $\mathrm{T}$ cell specificity, tumor immunogenicity and other costimulatory or inhibitory factors are not explicitly modeled but are summarized in the "killing probability". Also, the model does not explicitly include myeloid cells or other regulating factors for lymphocyte-mediated cytotoxicity. In the future, myeloid cells - and the crucial regulation of lymphocyte activity via macrophages (6) - could be incorporated into more complicated models. 
While these simplifications constrain the implications that can be drawn for certain molecular or signaling processes, it allows us to focus on the emergent features of the cellular interaction between key players in the tumor microenvironment. Most importantly, our model takes into account several quantitative histological observations and is thus partly calibrated with realworld tumors. Also, our model includes post-inflammatory fibrosis which gives rise to unexpected and informative emergent behavior. It therefore guides the possible translational developments, especially in selecting the ideal combination partners for synergistic clinical effects (Figure 6). This provides therefore a roadmap for an iterative enhancement of immunotherapy for immunologically "cold" tumors. 


\section{References}

1. Postow MA, Callahan MK, Wolchok JD. Immune Checkpoint Blockade in Cancer Therapy. J Clin Oncol 2015;33(17):1974-82 doi 10.1200/JCO.2014.59.4358.

2. Pardoll DM. The blockade of immune checkpoints in cancer immunotherapy. Nat Rev Cancer 2012;12(4):252-64 doi 10.1038/nrc3239.

3. Ribas A, Butterfield LH, Glaspy JA, Economou JS. Current developments in cancer vaccines and cellular immunotherapy. J Clin Oncol 2003;21(12):2415-32 doi 10.1200/JCO.2003.06.041.

4. Pure E, Lo A. Can Targeting Stroma Pave the Way to Enhanced Antitumor Immunity and Immunotherapy of Solid Tumors? Cancer Immunol Res 2016;4(4):269-78 doi 10.1158/2326-6066.CIR-16-0011.

5. Garrido-Laguna I, Hidalgo M. Pancreatic cancer: from state-of-the-art treatments to promising novel therapies. Nat Rev Clin Oncol 2015;12(6):319-34 doi 10.1038/nrclinonc.2015.53.

6. Halama N, Zoernig I, Berthel A, Kahlert C, Klupp F, Suarez-Carmona M, et al. Tumoral Immune Cell Exploitation in Colorectal Cancer Metastases Can Be Targeted Effectively by Anti-CCR5 Therapy in Cancer Patients. Cancer Cell 2016;29(4):587-601 doi 10.1016/j.ccell.2016.03.005.

7. 2017 American Cancer Society: https://www.cancer.org/cancer/colon-rectalcancer/detection-diagnosis-staging/survival-rates.html.

8. Fitzmaurice C, Allen C, Barber RM, Barregard L, Bhutta ZA, Brenner $\mathrm{H}$, et al. Global, Regional, and National Cancer Incidence, Mortality, Years of Life Lost, Years Lived With Disability, and Disability-Adjusted Life-years for 32 Cancer Groups, 1990 to 2015: A Systematic Analysis for the Global Burden of Disease Study. JAMA Oncol 2017;3(4):52448 doi 10.1001/jamaoncol.2016.5688.

9. Bonabeau E. Agent-based modeling: methods and techniques for simulating human systems. Proc Natl Acad Sci U S A 2002;99 Suppl 3:7280-7 doi 10.1073/pnas.082080899.

10. Farmer JD, Foley D. The economy needs agent-based modelling. Nature 2009;460(7256):685-6 doi 10.1038/460685a.

11. Epstein JM. Modelling to contain pandemics. Nature 2009;460(7256):687 doi 10.1038/460687a.

12. Conte R, Paolucci M. On agent-based modeling and computational social science. Front Psychol 2014;5:668 doi 10.3389/fpsyg.2014.00668.

13. Xu J, Vilanova G, Gomez H. Full-scale, three-dimensional simulation of early-stage tumor growth: The onset of malignancy. Computer Methods in Applied Mechanics and Engineering 2017;314:126-46 doi https://doi.org/10.1016/i.cma.2016.07.010.

14. Poleszczuk J, Macklin P, Enderling H. Agent-Based Modeling of Cancer Stem Cell Driven Solid Tumor Growth. Methods Mol Biol 2016;1516:335-46 doi 10.1007/7651_2016_346.

15. Poleszczuk J, Enderling H. Cancer Stem Cell Plasticity as Tumor Growth Promoter and Catalyst of Population Collapse. Stem Cells Int 2016;2016:3923527 doi 10.1155/2016/3923527. 
16. Waclaw B, Bozic I, Pittman ME, Hruban RH, Vogelstein B, Nowak MA. A spatial model predicts that dispersal and cell turnover limit intratumour heterogeneity. Nature 2015;525(7568):261-4 doi 10.1038/nature14971.

17. Paterson C, Nowak MA, Waclaw B. An exactly solvable, spatial model of mutation accumulation in cancer. Sci Rep 2016;6:39511 doi 10.1038/srep39511.

18. Jalalimanesh A, Shahabi Haghighi H, Ahmadi A, Soltani M. Simulation-based optimization of radiotherapy: Agent-based modeling and reinforcement learning. Math Comput Simul 2017;133:235-48 doi http://dx.doi.org/10.1016/j.matcom.2016.05.008.

19. Pourhasanzade F, Sabzpoushan SH, Alizadeh AM, Esmati E. An agent-based model of avascular tumor growth: Immune response tendency to prevent cancer development. Simulation 2017;1(17):1-17 doi 10.1177/0037549717699072.

20. López ÁG, Seoane JM, Sanjuán MAF. Destruction of solid tumors by immune cells. Commun Nonlinear Sci Numer Simul 2017;44:390-403 doi http://dx.doi.org/10.1016/j.cnsns.2016.08.020.

21. Lopez AG, Seoane JM, Sanjuan MA. A validated mathematical model of tumor growth including tumor-host interaction, cell-mediated immune response and chemotherapy. Bull Math Biol 2014;76(11):2884-906 doi 10.1007/s11538-014-0037-5.

22. Mallet DG, De Pillis LG. A cellular automata model of tumor-immune system interactions. J Theor Biol 2006;239(3):334-50 doi 10.1016/j.jtbi.2005.08.002.

23. Christophe $C$, Muller S, Rodrigues M, Petit AE, Cattiaux P, Dupre L, et al. A biased competition theory of cytotoxic T lymphocyte interaction with tumor nodules. PLoS One 2015;10(3):e0120053 doi 10.1371/journal.pone.0120053.

24. Lopez AG, Seoane JM, Sanjuan MAF. Dynamics of the cell-mediated immune response to tumour growth. Philos Trans A Math Phys Eng Sci 2017;375(2096) doi 10.1098/rsta.2016.0291.

25. Folcik VA, An GC, Orosz CG. The Basic Immune Simulator: an agent-based model to study the interactions between innate and adaptive immunity. Theor Biol Med Model 2007;4:39 doi 10.1186/1742-4682-4-39.

26. Dunn GP, Old $\mathrm{U}$, Schreiber RD. The immunobiology of cancer immunosurveillance and immunoediting. Immunity 2004;21(2):137-48 doi 10.1016/j.immuni.2004.07.017.

27. Halama N, Michel S, Kloor M, Zoernig I, Benner A, Spille A, et al. Localization and density of immune cells in the invasive margin of human colorectal cancer liver metastases are prognostic for response to chemotherapy. Cancer Res 2011;71(17):5670-7 doi 10.1158/0008-5472.CAN-11-0268.

28. Berthel A, Zoernig I, Valous NA, Kahlert C, Klupp F, Ulrich A, et al. Detailed resolution analysis reveals spatial $T$ cell heterogeneity in the invasive margin of colorectal cancer liver metastases associated with improved survival. Oncoimmunology 2017;6(3):e1286436 doi 10.1080/2162402X.2017.1286436.

29. Kather JN, Zollner FG, Schad LR, Melchers SM, Sinn HP, Marx A, et al. Identification of a characteristic vascular belt zone in human colorectal cancer. PLoS One 2017;12(3):e0171378 doi 10.1371/journal.pone.0171378.

30. Kather JN, Marx A, Reyes-Aldasoro CC, Schad LR, Zollner FG, Weis CA. Continuous representation of tumor microvessel density and detection of angiogenic hotspots in 
Author Manuscript Published OnlineFirst on September 18, 2017; DOI: 10.1158/0008-5472.CAN-17-2006

Author manuscripts have been peer reviewed and accepted for publication but have not yet been edited.

histological whole-slide images. Oncotarget 2015;6(22):19163-76 doi 10.18632/oncotarget.4383.

31. Breart B, Lemaitre F, Celli S, Bousso P. Two-photon imaging of intratumoral CD8+ T cell cytotoxic activity during adoptive T cell therapy in mice. J Clin Invest 2008;118(4):1390-7 doi $10.1172 / \mathrm{JCl} 34388$.

32. Boldajipour B, Nelson A, Krummel MF. Tumor-infiltrating lymphocytes are dynamically desensitized to antigen but are maintained by homeostatic cytokine. $\mathrm{JCl}$ Insight 2016;1(20):e89289 doi 10.1172/jci.insight.89289.

33. Berencsi $K$, Rani $P$, Zhang $T$, Gross L, Mastrangelo M, Meropol NJ, et al. In vitro migration of cytotoxic T lymphocyte derived from a colon carcinoma patient is dependent on CCL2 and CCR2. J Transl Med 2011;9:33 doi 10.1186/1479-5876-9-33.

34. Jiang $Y$, Li Y, Zhu B. T-cell exhaustion in the tumor microenvironment. Cell Death Dis 2015;6:e1792 doi 10.1038/cddis.2015.162.

35. Desmouliere A, Guyot C, Gabbiani G. The stroma reaction myofibroblast: a key player in the control of tumor cell behavior. Int J Dev Biol 2004;48(5-6):509-17 doi 10.1387/ijdb.041802ad.

36. Mahadevan D, Von Hoff DD. Tumor-stroma interactions in pancreatic ductal adenocarcinoma. Mol Cancer Ther 2007;6(4):1186-97 doi 10.1158/1535-7163.MCT-060686.

37. Coulson-Thomas VJ, Coulson-Thomas YM, Gesteira TF, de Paula CA, Mader AM, Waisberg J, et al. Colorectal cancer desmoplastic reaction up-regulates collagen synthesis and restricts cancer cell invasion. Cell Tissue Res 2011;346(2):223-36 doi 10.1007/s00441-011-1254-y.

38. Elmore S. Apoptosis: a review of programmed cell death. Toxicol Pathol 2007;35(4):495516 doi 10.1080/01926230701320337.

39. Mempel TR, Pittet MJ, Khazaie K, Weninger W, Weissleder R, von Boehmer $\mathrm{H}$, et al. Regulatory $T$ cells reversibly suppress cytotoxic $T$ cell function independent of effector differentiation. Immunity 2006;25(1):129-41 doi 10.1016/j.immuni.2006.04.015.

40. Caramalho I, Faroudi M, Padovan E, Muller S, Valitutti S. Visualizing CTL/melanoma cell interactions: multiple hits must be delivered for tumour cell annihilation. J Cell Mol Med 2009;13(9B):3834-46 doi 10.1111/j.1582-4934.2008.00586.x.

41. Eisenhauer EA, Therasse P, Bogaerts J, Schwartz LH, Sargent D, Ford R, et al. New response evaluation criteria in solid tumours: revised RECIST guideline (version 1.1). Eur J Cancer 2009;45(2):228-47 doi 10.1016/j.ejca.2008.10.026.

42. Cancer Genome Atlas N. Comprehensive molecular characterization of human colon and rectal cancer. Nature 2012;487(7407):330-7 doi 10.1038/nature11252.

43. Charoentong P, Finotello F, Angelova M, Mayer C, Efremova M, Rieder D, et al. Pancancer Immunogenomic Analyses Reveal Genotype-Immunophenotype Relationships and Predictors of Response to Checkpoint Blockade. Cell Rep 2017;18(1):248-62 doi 10.1016/j.celrep.2016.12.019.

44. Chen DS, Mellman I. Elements of cancer immunity and the cancer-immune set point. Nature 2017;541(7637):321-30 doi 10.1038/nature21349. 
45. Brookes ST, Whitely E, Egger M, Smith GD, Mulheran PA, Peters TJ. Subgroup analyses in randomized trials: risks of subgroup-specific analyses; power and sample size for the interaction test. J Clin Epidemiol 2004;57(3):229-36 doi 10.1016/j.jclinepi.2003.08.009.

46. Watt J, Kocher HM. The desmoplastic stroma of pancreatic cancer is a barrier to immune cell infiltration. Oncoimmunology 2013;2(12):e26788 doi 10.4161/onci.26788.

47. Hartmann N, Giese NA, Giese T, Poschke I, Offringa R, Werner J, et al. Prevailing role of contact guidance in intrastromal T-cell trapping in human pancreatic cancer. Clin Cancer Res 2014;20(13):3422-33 doi 10.1158/1078-0432.CCR-13-2972.

48. Kammertoens T, Schuler T, Blankenstein T. Immunotherapy: target the stroma to hit the tumor. Trends Mol Med 2005;11(5):225-31 doi 10.1016/j.molmed.2005.03.002.

49. Halvorsen TB, Seim E. Association between invasiveness, inflammatory reaction, desmoplasia and survival in colorectal cancer. J Clin Pathol 1989;42(2):162-6.

50. Galon J, Costes A, Sanchez-Cabo F, Kirilovsky A, Mlecnik B, Lagorce-Pages C, et al. Type, density, and location of immune cells within human colorectal tumors predict clinical outcome. Science 2006;313(5795):1960-4 doi 10.1126/science.1129139.

51. Mesker WE, Junggeburt JM, Szuhai K, de Heer P, Morreau H, Tanke HJ, et al. The carcinoma-stromal ratio of colon carcinoma is an independent factor for survival compared to lymph node status and tumor stage. Cell Oncol 2007;29(5):387-98.

52. Ozdemir BC, Pentcheva-Hoang T, Carstens JL, Zheng X, Wu CC, Simpson TR, et al. Depletion of carcinoma-associated fibroblasts and fibrosis induces immunosuppression and accelerates pancreas cancer with reduced survival. Cancer Cell 2014;25(6):719-34 doi 10.1016/j.ccr.2014.04.005.

53. Riedel A, Shorthouse D, Haas L, Hall BA, Shields J. Tumor-induced stromal reprogramming drives lymph node transformation. Nat Immunol 2016;17(9):1118-27 doi 10.1038/ni.3492. 


\section{Acknowledgements}

The authors would like to thank Anita Heinzelmann, Rosa Eurich and Jana Wolf (National Center for Tumor Diseases, Heidelberg, Germany), Katrin Wolk (University Medical Center Mannheim, Mannheim, Germany) and Nina Wilhelm (NCT Biobank, National Center for Tumor diseases, Heidelberg, Germany) for expert technical assistance. The authors are very grateful to Dr. Charles Neu (University Hospital Jena, Jena, Germany) for proofreading the article.

The results shown here are in part based upon data generated by the TCGA Research Network: http://cancergenome.nih.gov/.

\section{Potential conflict of interest}

The authors are not aware of any potential conflicts of interest.

\section{Figures}

Figure 1: Flowchart of the algorithm and visualization: an agent based model that generates realistic tumor nodules. (A) In our agent-based model, tumor cell agents and immune cell agents occupy a rectangular grid and can successively act. All cells migrate, divide, idle or die with fixed probabilities. Immune cells show a tendency to migrate towards tumor cells and can kill them. Immune cells that have killed five times become exhausted and can induce fibrosis. Tumor cells conglomerates can become necrotic. This process is repeated $500-5000$ times. Intermediary steps for accurate time scale discretization are not shown. (B) Tumor cells are shown in red and shaded according to their remaining proliferative potential. Immune cells are shown in blue and shaded according to their remaining kills. By default, fibrosis (stroma) is almost completely impermeable to all cells and shown in yellow, while necrosis is permeable to immune cells and shown in black. Scale bar: $2 \mathrm{~mm}$.

Figure 2: The model reproduces major immunological phenotypes of tumors. In general, four types of immune phenotypes can be distinguished: hot and cold tumors, immune excluded or eradicated tumors (fibrous scar). The agent-based model yields all those four phenotypes, depending only on the variation of two parameters (related to fibrosis generation and tumor cell killing). (A) cold tumor, (B) hot tumor, (C) immune excluded phenotype, (D) tumor eradication. Scale bars: $2 \mathrm{~mm}$. 
Figure 3: Stroma slows tumor growth in a lymphocyte-deprived, but mediates immune escape in a lymphocyte enriched environment. (A) experimental design, (B) tumor mass over time for all four groups, depending on probability of stroma generation (Stro low vs. high) and the magnitude of immune cell influx (Lym low vs. high) (C) outcome three months after baseline (60 days, dashed line). Response criteria were chosen analogous to the RECIST criteria (41). The subgroup "Stro low, Lym high" had a $100 \%$ response rate, the majority complete responses (i.e. eradication of all tumor cells). Abbreviations: $\mathrm{PD}=$ progressive disease, $\mathrm{SD}=$ stable disease, $\mathrm{PR}=$ partial remission, $\mathrm{CR}=$ complete remission.

Figure 4: High lymphocyte number and low stroma define a subgroup with longer survival in TCGA colorectal cancer patients. Data from $\mathrm{N}=261$ patients, publicly available through records from the TCGA database. (A) Hazard ratios are shown for overall survival together with 95\% confidence intervals and are adjusted for TNM status. (B) Kaplan-Meier plot for all four strata (product-limit survival estimates, number at risk shown below).

Figure 5: Increased stromal permeability can lead to tumor hyperprogression or eradication, depending on the immune cell number. (A) experimental procedure, (B) outcome in comparison to baseline by varying degrees of immune boosts and stromal permeability changes. Vertical axis from bottom to top: baseline, then 2-fold, 4-fold and 8-fold immune boost. Horizontal axis from left to right: stromal permeability baseline, then increase by $4 \%, 8 \%$ and $16 \%$ (100\% being completely permeable). $\mathrm{N}=24$ technical replicates per group. Stromal permeabilization leads to increased tumor progression, but enables tumor eradication if combined with an 8-fold immune boost.

Figure 6: Proposed model of combination effects of immunotherapy and stromal-targeted therapy: stromal depletion or permeability increase can lead to different outcomes. Our model suggests a crucial combination effect of stromal-targeting and immune-enhancing therapy. Activation of the adaptive immune response alone can stop tumor growth but cannot eradicate tumors. Stromal depletion or permeabilization alone benefits the tumor cells and leads to hyperprogression. Only the combination of stromal permeabilization and immune boosting enables tumor eradication. 


\section{Tables}

\begin{tabular}{|c|c|}
\hline Assumption & Ref. \\
\hline All cells can migrate, proliferate and die. & trivial \\
\hline $\begin{array}{l}\text { Tumor cells are composed of stem cells and non-stem cells. Stem cells can divide } \\
\text { symmetrically with a fixed probability. }\end{array}$ & (14) \\
\hline $\begin{array}{l}\text { Stem cells can proliferate indefinitely, all other cells die after a fixed number of } \\
\text { proliferation cycles. }\end{array}$ & (14) \\
\hline All cells can spontaneously enter apoptosis. & own data \\
\hline Tumor cells can spontaneously enter necrosis & own data \\
\hline $\begin{array}{l}\text { Tumor cells that are far from the outer margin have a higher probability of en- } \\
\text { tering necrosis than those cells closer to the margin. }\end{array}$ & own data \\
\hline $\begin{array}{l}\text { Immune cells are generated through a steady influx into the domain and prolif- } \\
\text { eration within the domain. }\end{array}$ & $\begin{array}{l}\text { (32), } \\
\text { own data }\end{array}$ \\
\hline $\begin{array}{l}\text { Immune cells move by a "random walk" but have a tendency to migrate towards } \\
\text { tumor cells. }\end{array}$ & $\begin{array}{l}\text { (31-33), } \\
\text { own data }\end{array}$ \\
\hline $\begin{array}{l}\text { Immune cells can kill adjacent tumor cells whenever they are close enough. Kill- } \\
\text { ing, like other events in the model, occurs stochastically with a fixed probability } \\
\text { and is not regulated by other factors. }\end{array}$ & (23) \\
\hline $\begin{array}{l}\text { Immune cells can kill five times before they become exhausted, which means } \\
\text { that they cannot kill anymore but can still proliferate. }\end{array}$ & $(23,34)$ \\
\hline $\begin{array}{l}\text { Activated immune cells give rise to stroma through a desmoplastic reaction } \\
\text { (stroma reaction). For simplicity, this behavior is restricted to immune cells that } \\
\text { have successfully killed five times in the model. }\end{array}$ & $(35,36)$ \\
\hline $\begin{array}{l}\text { By default, cells cannot migrate through stroma, but stromal permeability can } \\
\text { be increased optionally. }\end{array}$ & (37) \\
\hline
\end{tabular}

Table 1: Assumptions for the model and references for each assumption. For each assumption, one or more supporting references are listed. "Own data" refers to histological and wet-lab experiments that are part of this study. 


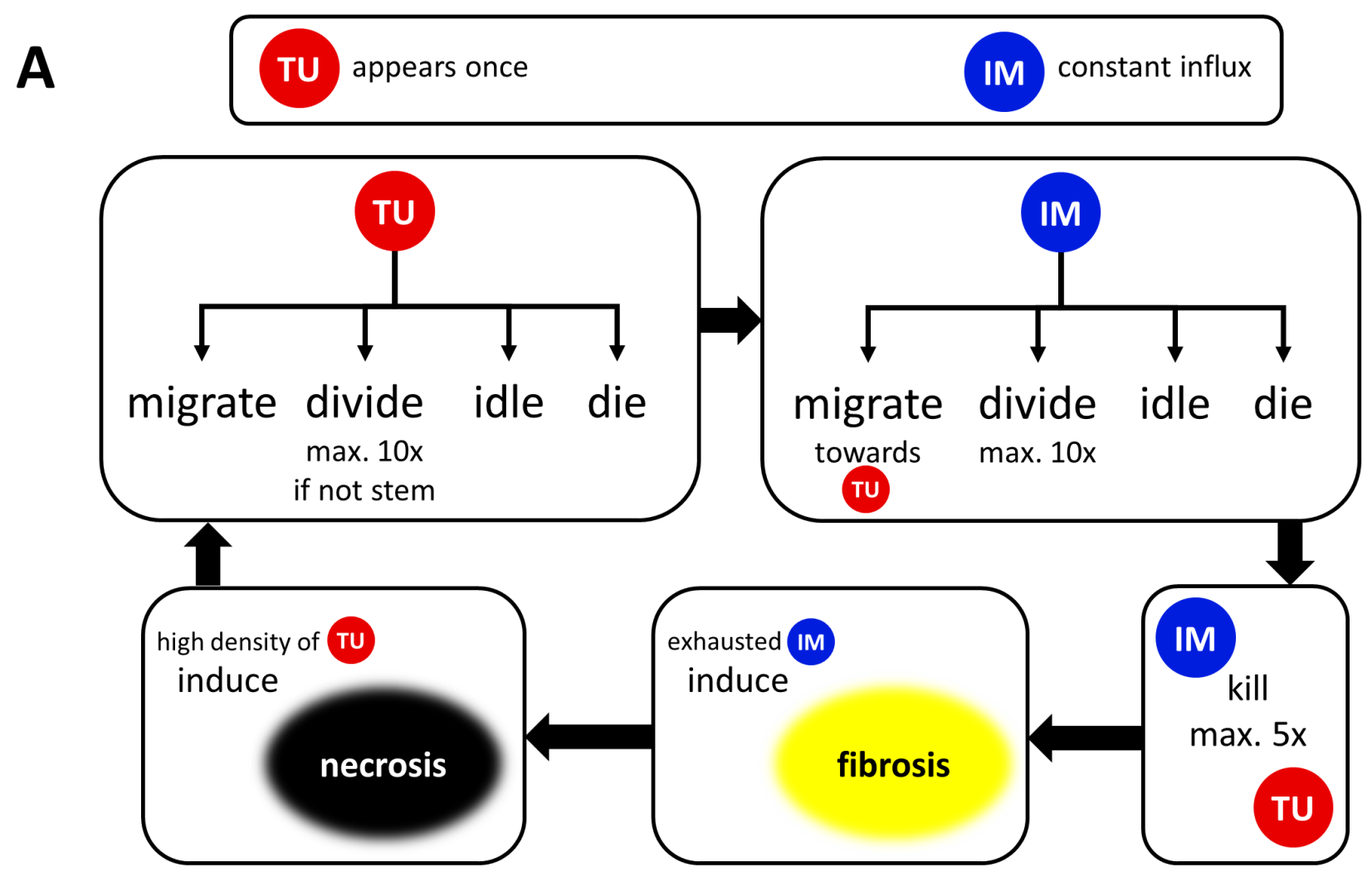

B

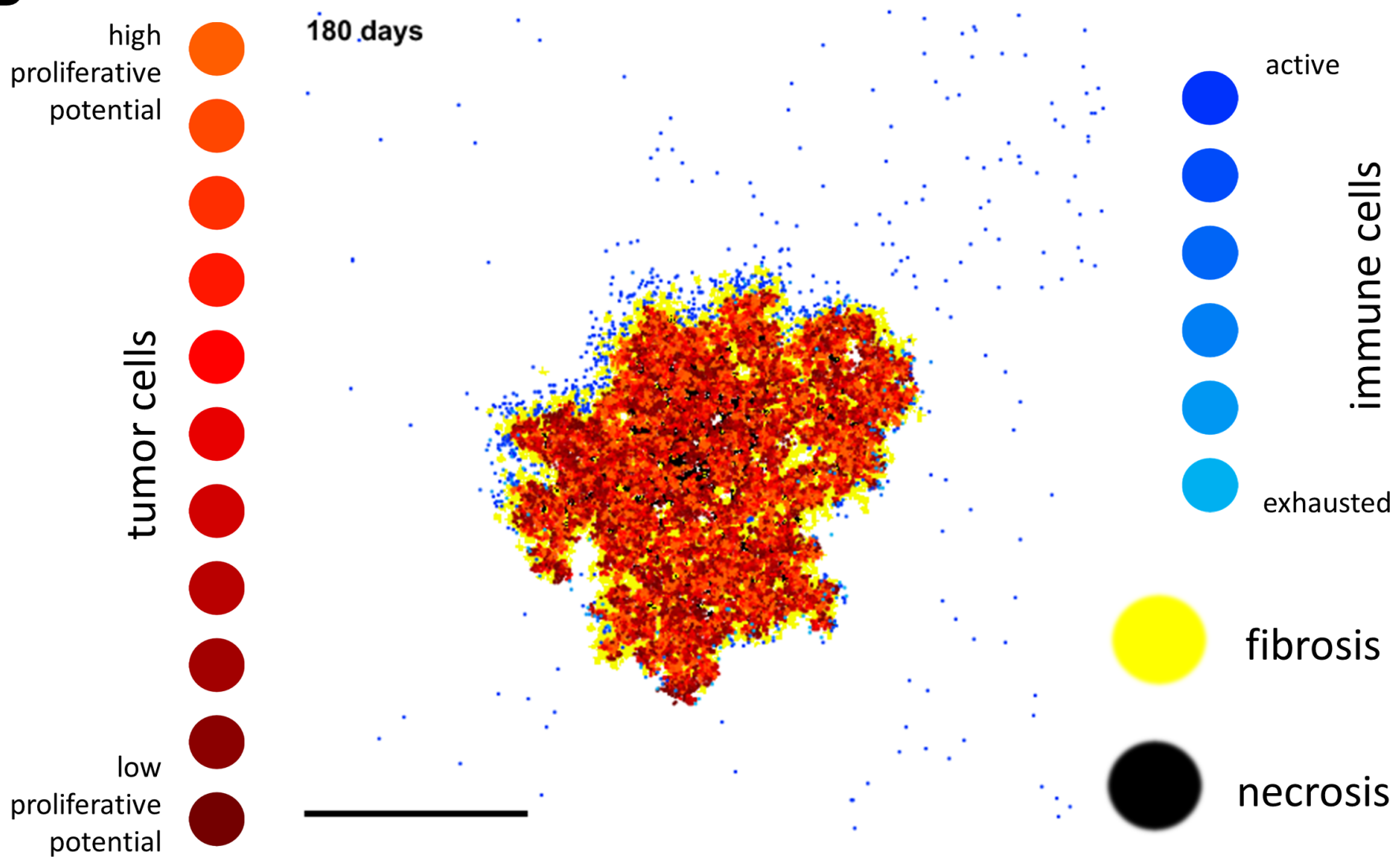

Figure 1 
A

175 days

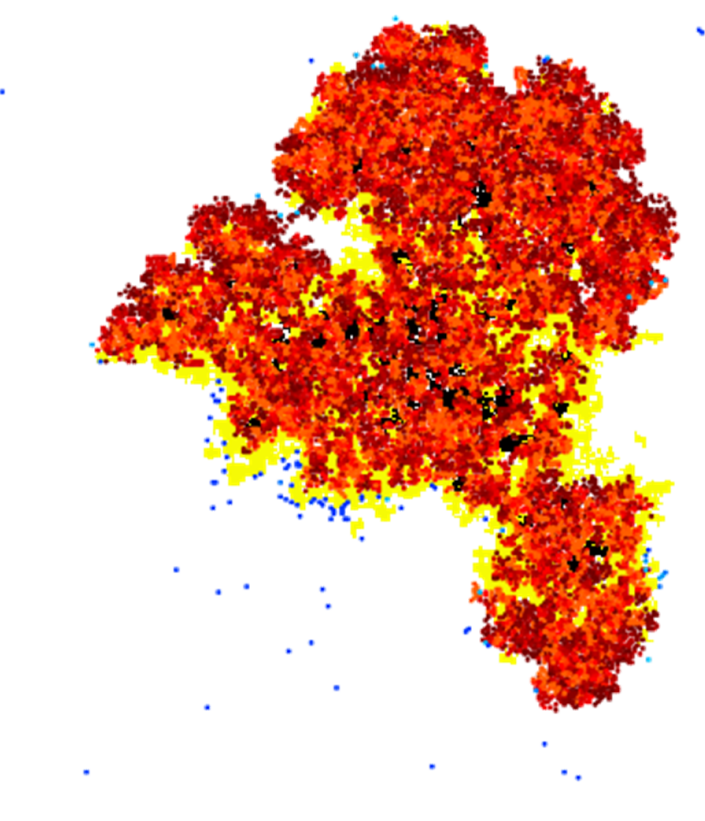

immune excluded

175 days

\section{Tumor Immune}

B

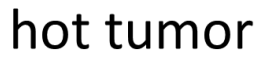

175 days

eradicated

175 days

\section{Necrosis}

Figure 2 

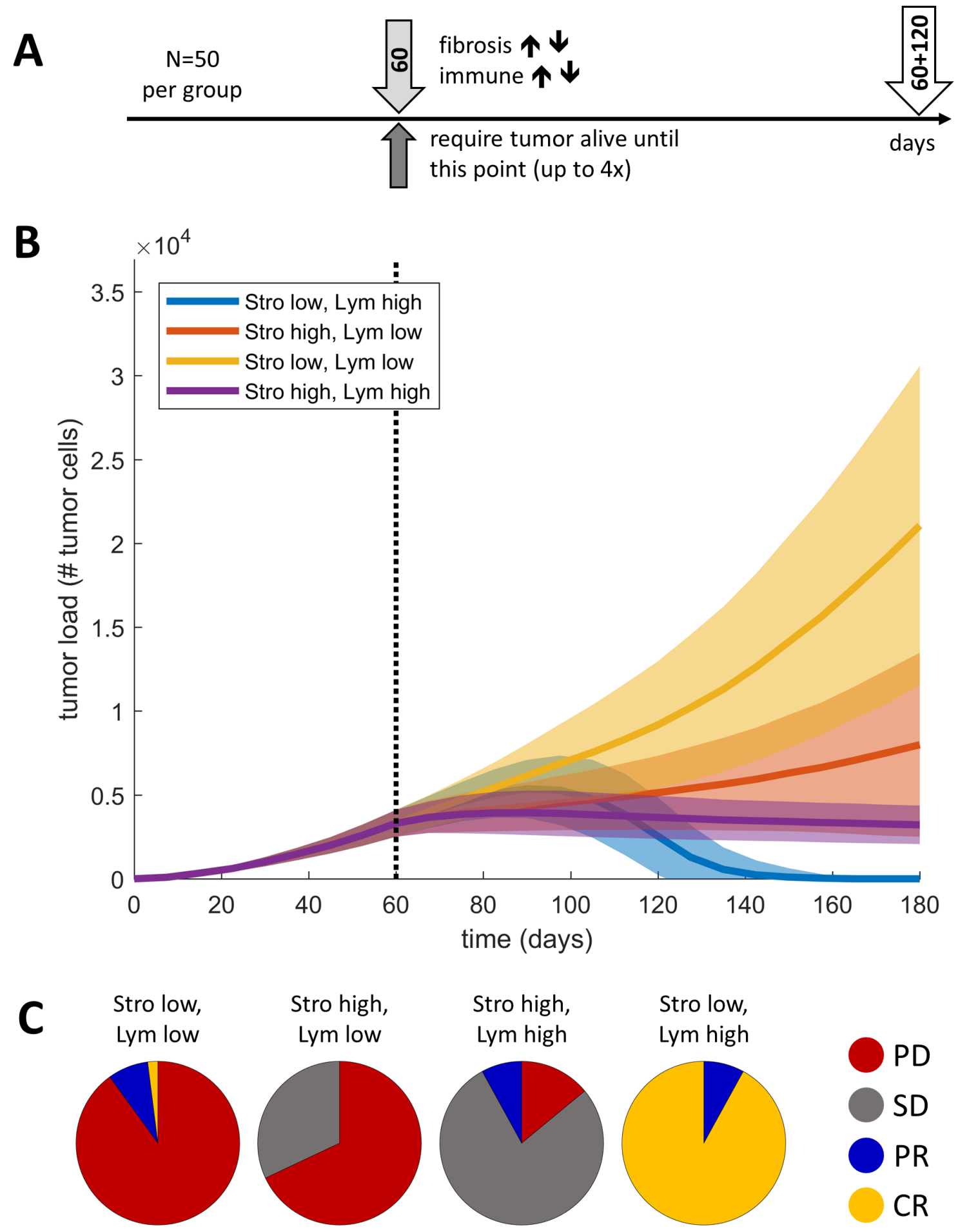

Figure 3 


\section{Hazard Ratios adjusted for TNM}

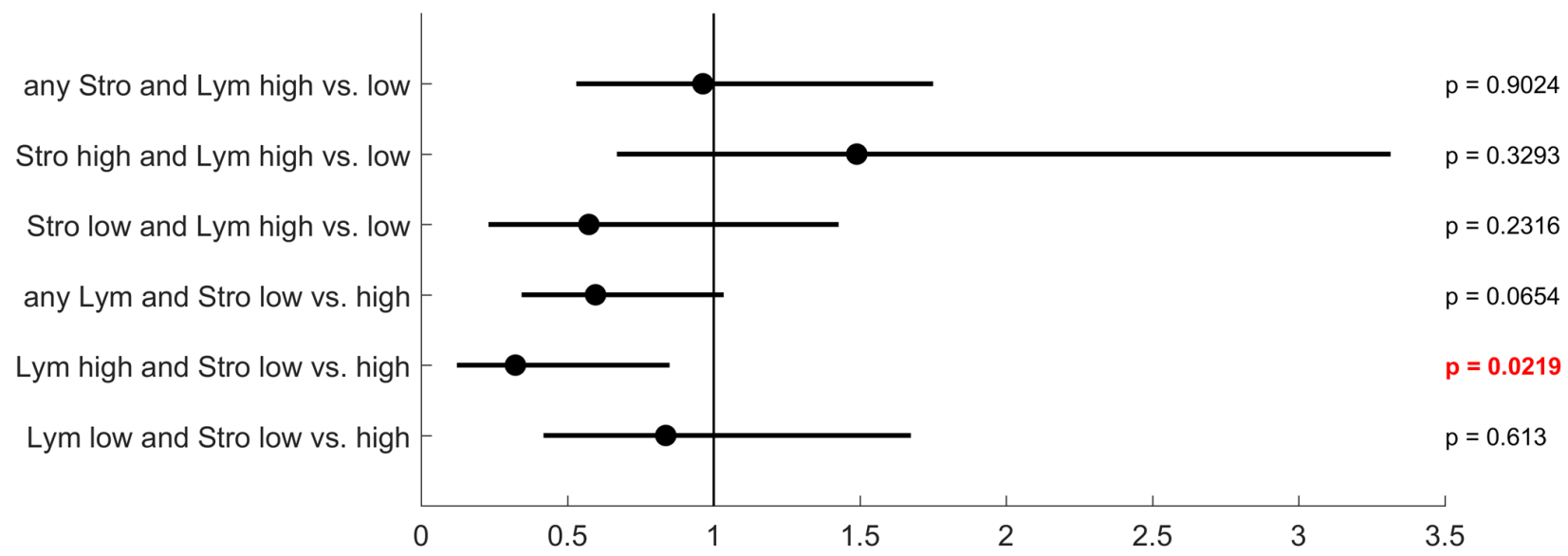

B

Kaplan-Meier curves for all groups

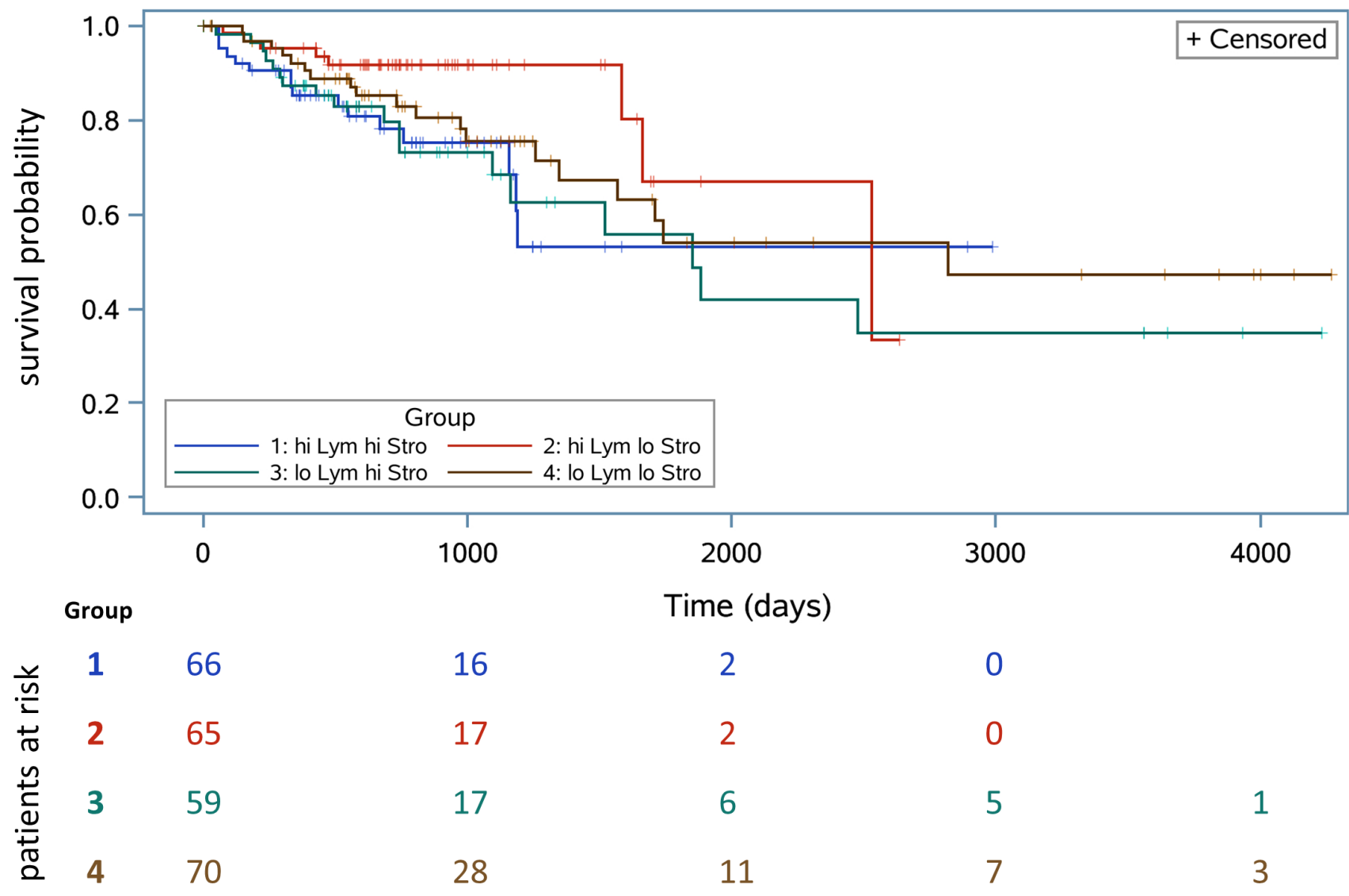

Figure 4 


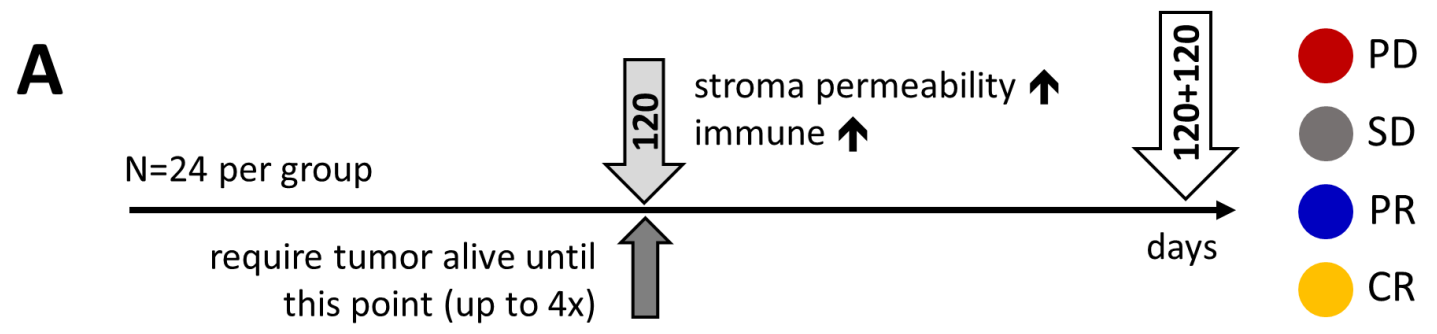

B

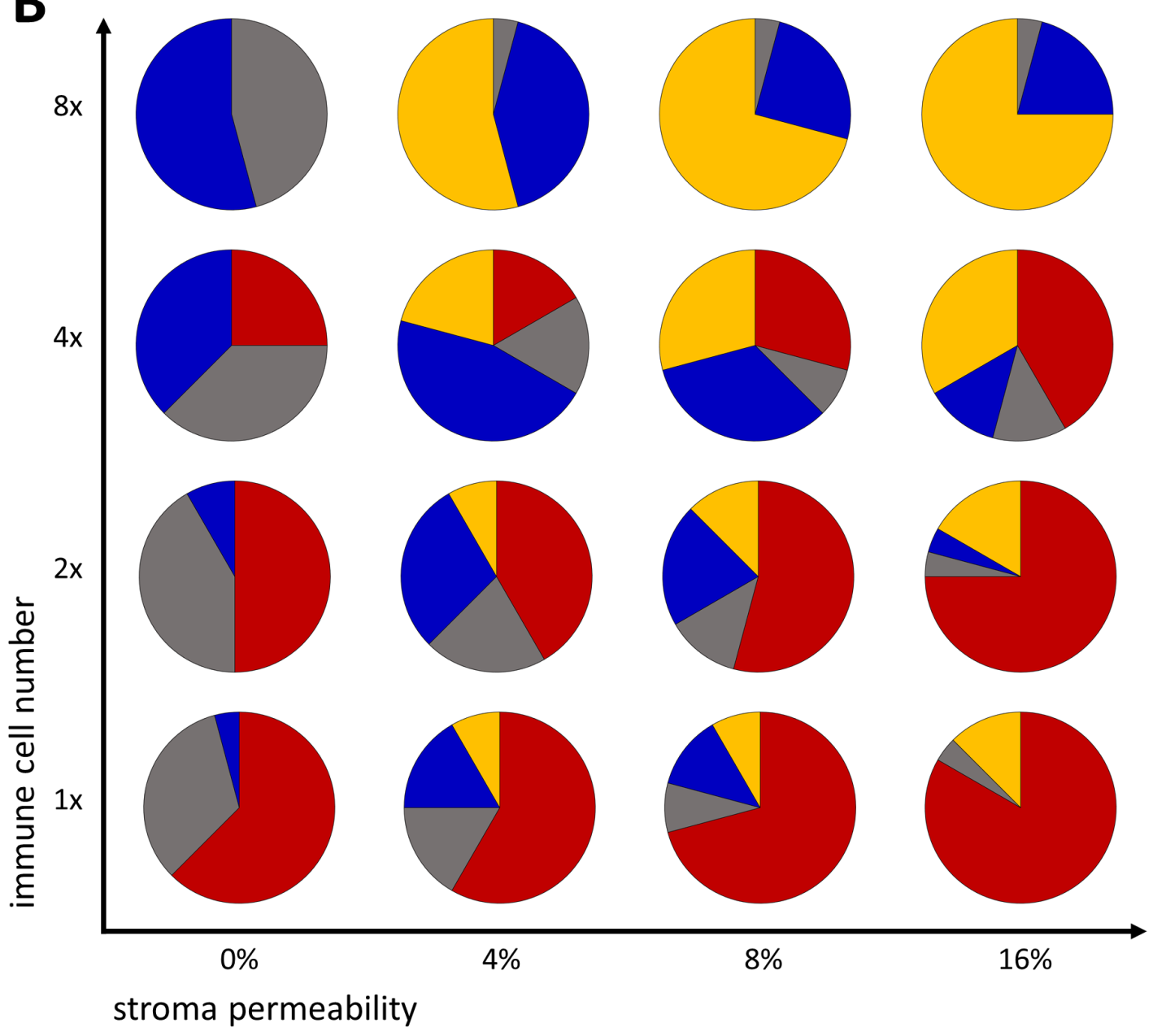

Figure 5 


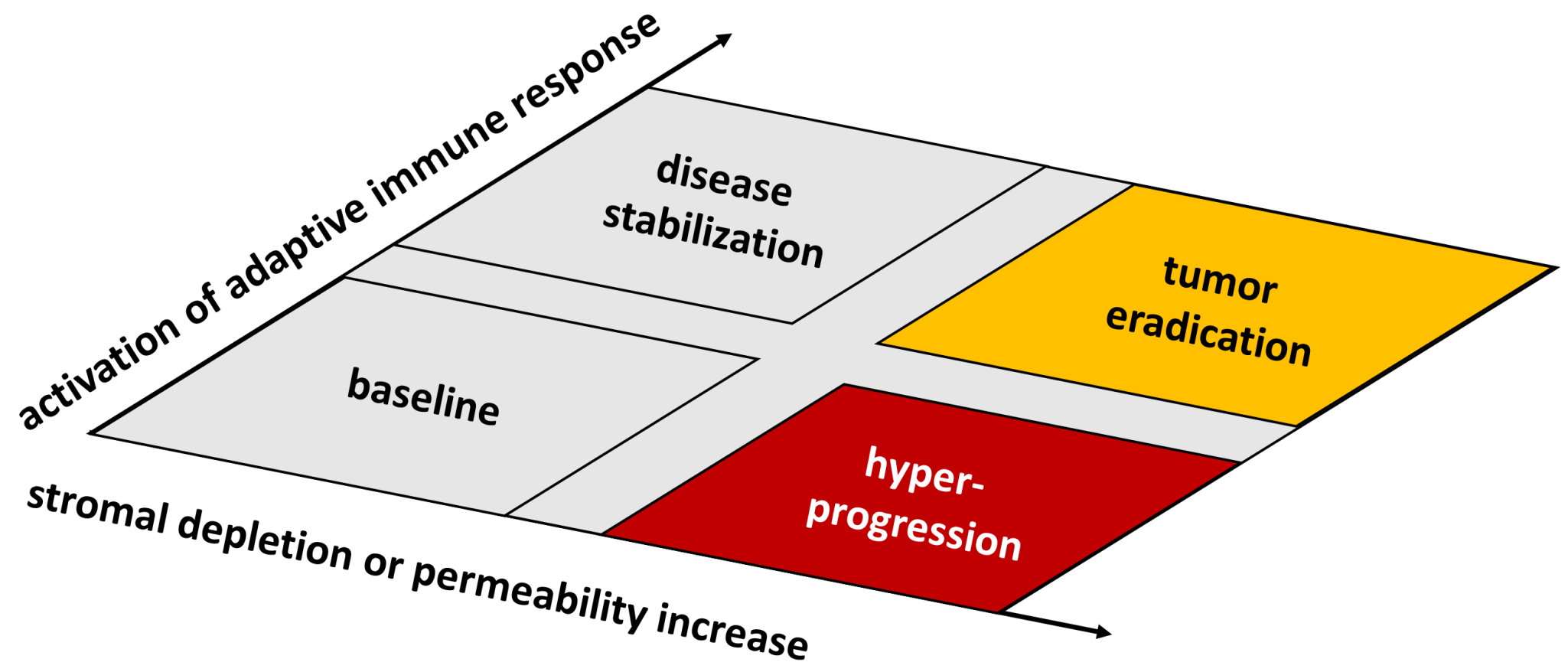

Figure 6 


\section{In silico modeling of immunotherapy and stroma-targeting therapies in human colorectal cancer}

Jakob Nikolas Kather, Jan Poleszczuk, Meggy Suarez-Carmona, et al.

Cancer Res Published OnlineFirst September 18, 2017.

Updated version Access the most recent version of this article at: doi:10.1158/0008-5472.CAN-17-2006

Supplementary Access the most recent supplemental material at:

Material http://cancerres.aacrjournals.org/content/suppl/2017/09/16/0008-5472.CAN-17-2006.DC1

Author Author manuscripts have been peer reviewed and accepted for publication but have not yet been Manuscript edited.

\section{E-mail alerts Sign up to receive free email-alerts related to this article or journal.}

Reprints and Subscriptions

Permissions
To order reprints of this article or to subscribe to the journal, contact the AACR Publications Department at pubs@aacr.org.

To request permission to re-use all or part of this article, use this link http://cancerres.aacrjournals.org/content/early/2017/09/16/0008-5472.CAN-17-2006.

Click on "Request Permissions" which will take you to the Copyright Clearance Center's (CCC) Rightslink site. 